1 Version Date: July 27, 2020

\title{
Comparative Performance of Popular Methods for Hybrid Detection using Genomic Data
}

\author{
Sungsik Kong ${ }^{1}$ And Laura S. Kubatko ${ }^{1,2, *}$ \\ ${ }^{1}$ Department of Evolution, Ecology, and Organismal Biology, The Ohio State University, Columbus, OH \\ 43210, USA \\ 2 Department of Statistics, The Ohio State University, Columbus, OH 43210, USA \\ ${ }^{*}$ Correspondence to be sent: Department of Statistics, Department of Evolution, Ecology, and Organismal \\ Biology, The Ohio State University, Columbus, OH 43210,USA; E-mail: lkubatko@stat.osu.edu
}

\begin{abstract}
3 Interspecific hybridization is an important evolutionary phenomenon that generates 4 genetic variability in a population and fosters species diversity in nature. The availability ${ }_{5}$ of large genome scale datasets has revolutionized hybridization studies to shift from the ${ }_{6}$ examination of the presence or absence of hybrids in nature to the investigation of the 7 genomic constitution of hybrids and their genome-specific evolutionary dynamics.

8 Although a handful of methods have been proposed in an attempt to identify hybrids, 9 accurate detection of hybridization from genomic data remains a challenging task. The available methods can be classified broadly as site pattern frequency based and population genetic clustering approaches, though the performance of the two classes of methods under different hybridization scenarios has not been extensively examined. Here, we use simulated data to comparatively evaluate the performance of four tools that are commonly used to infer hybridization events: the site pattern frequency based methods $H y D e$ and the $D$-statistic (i.e., the ABBA-BABA test), and the population clustering approaches
\end{abstract}


structure and ADMIXTURE. We consider single hybridization scenarios that vary in the time of hybridization and the amount of incomplete lineage sorting (ILS) for different proportions of parental contributions $(\gamma)$; introgressive hybridization; multiple hybridization scenarios; and a mixture of ancestral and recent hybridization scenarios. We focus on the statistical power to detect hybridization, the false discovery rate (FDR) for the $D$-statistic and $H y D e$, and the accuracy of the estimates of $\gamma$ as measured by the mean squared error for HyDe, structure, and ADMIXTURE. Both HyDe and the D-statistic demonstrate a high level of detection power in all scenarios except those with high ILS, although the $D$-statistic often has an unacceptably high FDR. The estimates of $\gamma$ in $H y D e$ are impressively robust and accurate whereas structure and ADMIXTURE sometimes fail to identify hybrids, particularly when the proportional parental contributions are asymmetric (i.e., when $\gamma$ is close to 0). Moreover, the posterior distribution estimated using structure exhibits multimodality in many scenarios, making interpretation difficult.

Our results provide guidance in selecting appropriate methods for identifying hybrid populations from genomic data.

Key words:

The ABBA-BABA test, ADMIXTURE, Patterson's D-statistic, Hybridization, HyDe, Introgression, Structure.

$36 \mathrm{p}$

37 (1) of addition, phenotypes can sometimes appear to be counterintuitive. For example, heterosis or transgressive segregation, where the phenotype of the hybrid falls outside of the range of 
parental variation, and adaptive introgression, where the hybrid closely resembles one of the parents via backcrossing (see review by Goulet et al., 2017), are cases in which hybrid detection would be challenging using phenotypic data alone.

The availability of ever-increasing amounts of genomic data coupled with advances in statistical and computational methods for handling large datasets has enabled researchers to use genomic data for detecting signals of hybrid ancestry in the past history of a species (Twyford and Ennos, 2012). While heterogeneity in the gene tree distribution across the genome of a hybrid achieved by genomic mosaicism (Folk et al., 2018) is very useful for this task, other evolutionary processes may result in similar genomic footprints, including incomplete lineage sorting (ILS), horizontal gene transfer, recombination, gene duplication and loss, and historical nonrandom mating, among others (Maddison, 1997). Much methodological work has been devoted to detecting hybridization in the presence of ILS as both processes are likely to occur simultaneously (Holder et al., 2001; Joly et al., 2009; Kubatko, 2009; Meng and Kubatko, 2009; Solís-Lemus and Ané, 2016). We briefly introduce several model-based methods that are popularly employed to detect hybridization.

Patterson's D-statistic (Green et al., 2010; Durand et al., 2011; Patterson et al., 2012), popularly referred to as the ABBA-BABA test, is widely used to conduct a hypothesis test to infer introgression in spite of ILS. It is based on the magnitude of the observed difference in two discordant bi-allelic site pattern frequencies. The $D$-statistic is robust across a wide range of genetic distances although it may be sensitive to population size (Green et al., 2010; Jónsson et al., 2014; Martin et al., 2016; Kumar et al., 2017; Zheng and Janke, 2018). Refinements of the method continue to be explored in recent studies, including extensions that allow for application of the test to cases beyond the four-taxon phylogeny as well as those in which multiple individuals per population are sampled (Pease and Hahn, 2015; Elworth et al., 2018; Soraggi et al., 2018; Hibbins and Hahn, 2019; Malinsky, 2019). 
Kubatko and Chifman (2019) proposed a method based on phylogenetic invariants for detecting both recent and ancestral hybridization events that considers both ILS and hybrid speciation within the same framework. Note that hybrid speciation differs from introgression as the taxon resulting from the process of hybrid speciation is maintained as a distinct taxonomic entity, whereas in the case of introgression, the hybrid entity merges with one of the parents via repeated backcrossing. The two processes will thus result in different topologies, as shown in Figure 1a (hybrid speciation) and 1b (introgression). Implemented in the computer program HyDe (Blischak et al., 2018), this method can examine genomic-scale data for a large number of taxa and detect the population(s) that may have arisen via hybrid speciation as well as their putative parental populations with statistical power that is similar to the $D$-statistic (Kubatko and Chifman, 2019). In addition to conducting a formal statistical test for hybridization, $H y D e$ estimates the inheritance parameter, $\gamma$, that quantifies the genomic contributions of the parents to the hybrid and has been useful in detecting hybridization in frogs (Chan et al., 2019), Cichlid fish (Olave and Meyer, 2020), Persian Walnut (Zhang et al., 2019), and polyploid trees (Wagner et al., 2019). Because HyDe is a site pattern based method like the $D$-statistic, it is computationally efficient and not limited by the size of the dataset. However, genome-scale data may be necessary for optimal performance in complex hybridization scenarios (Blischak et al., 2018).

Population genetic clustering approaches are popularly employed to identify hybrid individuals from genetic data and to hypothesize historical processes that shaped present population structure. Often implemented within a maximum likelihood (ML; e.g., ADMIXTURE; Alexander et al., 2009) or Bayesian-inference (BI; e.g., structure; Pritchard et al., 2000) framework, these methods provide a statistical basis to estimate the contribution of various genetic groups to an individual's ancestry through estimation of probabilistic quantities called ancestry coefficients. These coefficients are interpreted as the proportion of genetic material inherited from ancestral gene pools to an individual in 
contemporary populations, but they are also often interpreted to be equivalent to the proportion of parental contributions in a hybrid population (Vähä and Primmer, 2005). There is no standardized and objective way to interpret these values, and most studies simply set an arbitrary probability threshold for use in classifying an individual as pure or admixed (Randi, 2008; Khosravi et al., 2013; Ito et al., 2015). Reliance on the ancestry coefficient can lead to mis- or overinterpretation of the processes in nature because different evolutionary scenarios can result in indistinguishable ancestry coefficients (Barilani et al., 2007; Anderson and Dunham, 2008; Lawson et al., 2018). Moreover, these methods are sensitive to the choice of markers, the level of genetic differentiation between populations, and the amount of data utilized (Vähä and Primmer, 2005; Latch et al., 2006; Kalinowski, 2011). Importantly, many of these methods were not originally designed to detect hybrid individuals, although they are popularly employed for this task.

The ability of the different population clustering methods to identify hybrids has previously been compared and evaluated. Two BI methods, structure and NewHybrids (Anderson and Thompson, 2002), were found to perform similarly well in detecting recent hybridization using simulated data with varying levels of genetic divergence and numbers of loci, although NewHybrids performed slightly better when the dataset contained a mix of $F_{1}$ hybrids and introgressants (Vähä and Primmer, 2005; Oliveira et al., 2015). Comparisons of structure, NewHybrids, BAPS (Corander and Marttinen, 2006) and GeneClass (Piry et al., 2004) using simulated hybrids showed that the former three full BI methods outperformed the semi-Bayesian approach employed by GeneClass (Sanz et al., 2009). However, all methods showed reduced efficiency in their ability to detect hybrids as the rate of backcrossing increased. Bohling et al. (2013) and Neophytou (2014) compared the performance of structure and BAPS and found that structure was more likely to detect admixed populations and accurately estimate the ancestry coefficients, although misclassification occasionally occurred. Interestingly, both methods were outperformed by an ML-based assignment program (the Canid Assignment Test; Bohling et al., 2013). 
In this study, we aim to comparatively evaluate the performance of four methods that are commonly used for hybrid detection using genomic data, $H y D e$, the $D$-statistic, structure, and ADMIXTURE, in a wide range of hybridization scenarios using simulated data. We consider six evolutionary scenarios: (1) a single hybridization event that varies in the time of hybridization and the amount of ILS for different values of $\gamma$; (2) introgression; (3) two and (4) three recent independent hybridization events; (5) three hybridization events that overlap (i.e., parental lineages are involved in more than one hybridization event); and (6) a mixture of ancestral and recent hybridization events. The evaluation is based on the power of the methods to detect hybridization and on the false discovery rates (FDR) for the $D$-statistic and HyDe, while the accuracy of estimated parental contributions is evaluated using mean squared error (MSE) for HyDe, structure, and ADMIXTURE.

MATERIALS AND Methods

\section{Data Simulation}

The six hybridization scenarios considered in this study are illustrated in Figure 1. For the single hybridization scenario (Fig. 1a), we used several different combinations of parameters: $\tau_{1} \in\{0.05,0.5,1.5,2.5,5\}$ with $\tau_{2}$ fixed at 1.5 (all branch lengths are measured in coalescent units, defined to be the number of $2 N$ generations, where $N$ is the effective population size) and $\gamma \in\{0,0.1,0.2,0.3,0.4,0.5\}$, producing 30 combinations; and $\tau_{1}$ fixed at 1.5 with $\tau_{2} \in\{0.05,0.5,2.5,5\}$ and $\gamma \in\{0,0.1,0.2,0.3,0.4,0.5\}$, leading to another 24 combinations. Larger values of $\tau_{1}$ lead to increases in the amount of time that has elapsed since hybridization, which allows time for mutations to accumulate in the descendant lineages, while smaller values of $\tau_{2}$ lead to increases in the amount of ILS. We chose 1.5 for the 'default' edge length as it will result in a modest amount of ILS (Degnan and Rosenberg, 2006). The edge that links the root and the most recent common ancestor 
a)

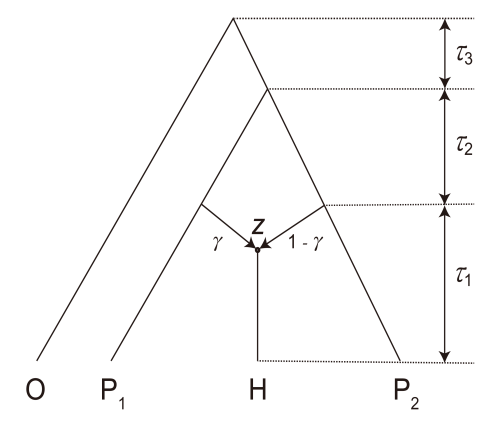

d)

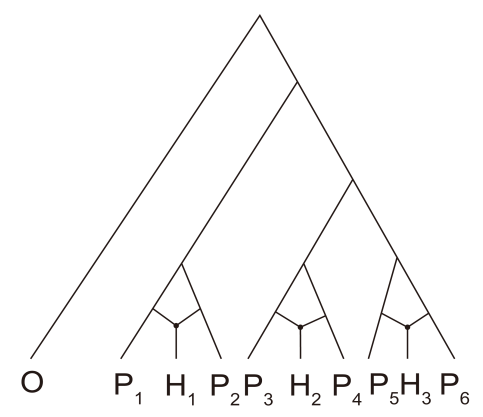

b)

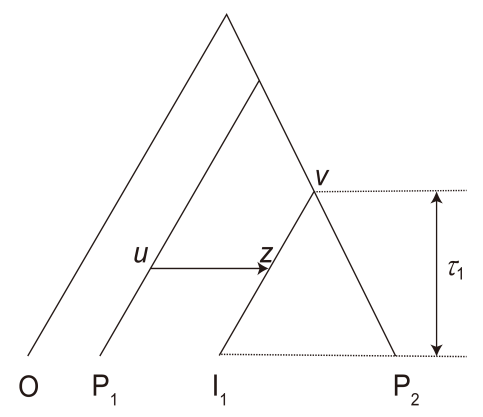

e)

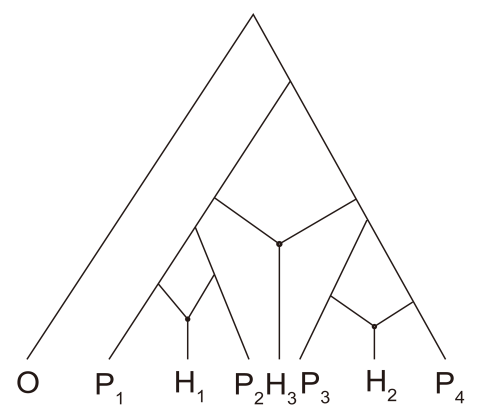

c)

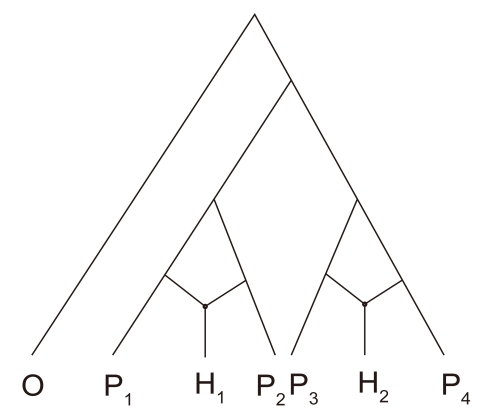

f)

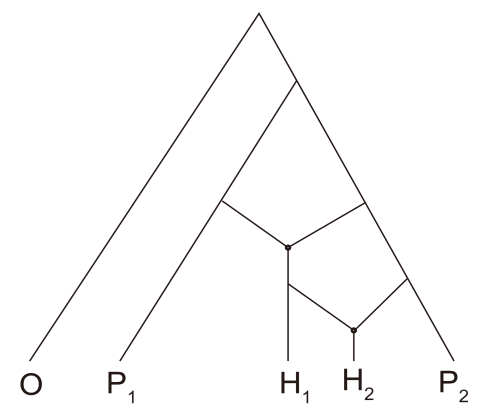

Fig. 1. Six hybridization scenarios considered in this study: a) single hybridization event, b) introgression, c) two independent hybridization events, d) three independent hybridization events, e) three hybridization events that overlap (i.e., parental lineages are involved in more than one hybridization event), and f) mixture of ancestral and recent hybridization events. The outgroup population is labelled as $\mathrm{O}$, parental populations are labelled as $\mathrm{P}_{i}$, and hybrids or introgressants are labelled as $\mathrm{H}_{i}$ or $\mathrm{I}_{i}$, respectively. Edge length, or the interval between speciation events, is denoted as $\tau_{i}$. Distinct populations and $\tau$ s are labelled with different integers in subscript $i$, and $\tau_{1}$ is more recent than $\tau_{2}$. In a), $\gamma$ and $1-\gamma$ below each hybrid edge denote the contribution of the corresponding parental population to the genome of the hybrid. The hybrid node is labelled as $z$ in a) and b). In b), the two parental tree nodes that contribute to the hybrid node $z$ are denoted by $u$ and $v$.

(MRCA) of the ingroup populations $\left(\tau_{3}\right)$ was set to 5.0 to ensure sufficient genomic differentiation of the outgroup. For the scenarios in Figure 1b-1f, we assigned a single set of parameters: $\gamma=0.5$ with all $\tau$ fixed to 1.5 except for the edge linked to the root and MRCA of the ingroup populations, which was set to 5.0.

For each condition, $10^{3}$ gene trees were simulated using $m s$ (Hudson, 2002). We additionally simulated $10^{4}$ and $10^{5}$ gene trees for the single hybridization scenario (Fig. 1a) when $\tau_{1}=0.05, \tau_{2}=1.5$, and $\gamma \in\{0,0.1,0.2,0.3,0.4,0.5\}$ to increase the size of datasets by a factor of ten and one hundred, respectively, to further evaluate the performance of HyDe when the level of ILS was very high. For the introgression scenario (Fig. 1b), we 
8

varied the height of node $u$ to be $6^{-1}, 6^{-2}, 6^{-3}, 6^{-4}$, and $6^{-5}$ of $\tau_{1}$ with

$\gamma \in\{0,0.1,0.2,0.3,0.4,0.5\}$ to evaluate the behavior of $H y D e$ when the timing of the introgression event varied. While $m s$ only takes input in a tree format and cannot be used to simulate gene trees directly from a network, we note that a network can be decomposed into a forest of trees since $r$ reticulations display up to $2^{r}$ trees that can be obtained by removing one of the two incoming hybrid edges for each hybrid node (denoted as $z$ in Fig. 1a and 1b). We generally followed the parameter settings in Gerard et al. (2011) and assumed an island model with no migration between equally-sized, non-recombining, and panmictic subpopulations following divergence. We used the $m s$ command $-\mathrm{j} t \mathrm{t} j$ that moves all lineages in subpopulation $i$ to subpopulation $j$ at time $t$ that corresponds to the desired $\tau$ for each tree. Each parent population contained 10 individuals, five individuals were assigned to the hybrid or introgressant population(s), and one to the outgroup population. Note that the branch lengths were halved when constructing the command blocks as $m s$ uses coalescent units of $4 N$ generations rather than the $2 N$ generations used for common models. Gene trees were simulated in proportion to the desired $\gamma$. For example, Figure 1a was decomposed into two trees and we simulated $10^{3} \gamma$ gene trees from one tree obtained from the model network

$$
\left(O: 5,\left(\left(P_{1}: \tau_{1}, H: \tau_{1}\right), P_{2}: \tau_{2}\right)\right)
$$

and $10^{3}(1-\gamma)$ from the other tree

$$
\left(O: 5,\left(P_{1}: \tau_{2},\left(H: \tau_{1}, P_{2}: \tau_{1}\right)\right)\right)
$$

Similarly, the networks in Figure 1b-1f were broken down into $2^{r}$ trees and the number of gene trees simulated for each tree was $10^{3} \times\left(2^{-r}\right)$.

The simulated gene trees were then used as input to simulate DNA sequences using seq-gen (Rambaut and Grassly, 1997) with a length of 100 base pairs per gene to mimic the short-read lengths generated by next-generation sequencing methods (Kubatko and Chifman, 2019). We scaled each branch of the input gene trees by setting the parameter $-\mathrm{s}$ 
as 0.036 , to make them equal to the expected number of substitutions per site for each branch (Rambaut and Grassly, 1997). The sequences were generated under the HKY model with $\kappa=1$, and A,C,G, and T frequencies of $0.300414,0.191363,0.196748$, and 0.311475, respectively, as described in Yu et al. (2014) and Solís-Lemus and Ané (2016).

The sequences were formatted using the concat function in goalign (https://github.com/fredericlemoine/goalign) followed by single-nucleotide polymorphism (SNP) extraction and format conversion into fasta, phylip, and vcf for subsequent analyses using snp-sites (Page et al., 2016). The SNP data were converted into structure readable format using PGDSpider (Lischer and Excoffier, 2012) and ADMIXTURE readable . bed format using plink2 (http://www.cog-genomics.org/plink/2.0/; Chang et al., 2015). In addition, we produced a truncated version of the simulated datasets that only contained one individual for each population as required for the computation of the $D$-statistic.

(1)

Fig. 1b). The outgroup defines the ancestral state (denoted as A) relative to the derived state $(\mathrm{B})$ where $\mathrm{A} \neq \mathrm{B} \in\{A, C, T, G\}$, thus the model tree that can be obtained by removing the reticulation edge between nodes $u$ and $z,\left(\left(\left(P_{2}, I_{1}\right), P_{1},\right) O\right)$, is supported by the site patterns BBAA or BBBA. Polyphyletic appearances of the derived state in the 
10

tree, $\mathrm{ABBA}$ and $\mathrm{BABA}$, represent discordant site patterns, as they are not expected in the model tree mentioned above but are expected for gene trees $\left(\left(\left(I_{1}, P_{1}\right), P_{2}\right), O\right)$ and $\left(\left(\left(P_{2}, P_{1}\right), I_{1}\right), O\right)$, respectively. These discordant site patterns are expected to occur with equal frequency if ILS is the sole source of conflict (Goulet et al., 2017). However, introgression between $I_{1}$ and either $P_{1}$ or $P_{2}$ will disproportionately increase the frequency of ABBA or BABA for each expected gene tree. This model assumes a single introgression event in the four-taxon network being tested and a single mutation per site with a negligible number of homoplasious substitutions. The $D$-statistic is calculated by:

$$
D=\frac{n_{A B B A}-n_{B A B A}}{n_{A B B A}+n_{B A B A}}
$$

where $n_{A B B A}$ represents the count of site pattern $\mathrm{ABBA}$ and $n_{B A B A}$ is the count of BABA sites.

Hybrid detection in $H y D e$ is based on phylogenetic invariants, which are polynomials in the site patterns that evaluate to zero on one tree topology but are non-zero for one or more other topologies (Felsenstein, 1991; Kubatko and Chifman, 2019). Consider two tree topologies (1) and (2) obtained from Figure 1a and two linear relationships of the site pattern counts that arise on the network:

$$
f_{1}=n_{A A B B}-n_{A B A B}
$$

$$
f_{2}=n_{A B B A}-n_{A B A B}
$$

In the absence of hybridization, we expect zero when $f_{1}$ is evaluated on the site-pattern counts for topology (1) and $f_{2}$ for topology (2). However, neither of them is zero when the site-pattern counts are evaluated on the network with $\gamma \in(0,1)$. These functions are very attractive because the ratio of their expectations is a function of $\gamma$ :

$$
\frac{f_{1}}{f_{2}}=\frac{\gamma}{1-\gamma}
$$

We note that this ratio is zero when $\gamma=0$, which is used as the basis for the hypothesis test in $H y D e$. 
Two input files are required to run $H y D e$ : a multiple sequence alignment that contains at least one outgroup and three or more ingroup populations in phylip format, and a map file, a two-column text table with individual names in the first column and the name of the population to which each individual belongs in the second column. The order of the individuals in the map file must match the order of individuals in the phylip alignment. Because the order in the phylip file produced by our simulation pipeline changes in every replicate, the corresponding map file must be re-created in every run, which we automated using a custom script. The $D$-statistic was calculated on the truncated dataset using $\mathrm{ABBA}$ and $\mathrm{ABAB}$ counts reported in the $H y D e$ output using a custom $R$ script. We used ABAB instead of BABA because the order in which HyDe counts the site patterns is to list the ingroup individuals first followed by the outgroup, as opposed to the model for the $D$-statistic, which lists the outgroup first. In some cases where position of $\mathrm{P}_{1}$ and $\mathrm{P}_{2}$ were switched, particularly when $\gamma=0$, we used the frequency of AABB instead of ABBA, as the tree assumed in this case would be $\left(O,\left(\left(P_{2}, H\right), P_{1}\right)\right)$ instead of topology (2). While the $z$-score is often used to assess significance since $z \geqslant 3$ corresponds to $p<0.0013$ (Zheng and Janke, 2018), an extra step to compute the $p$-value was conducted to allow objective comparisons with the $p$-value in the output of $H y D e$.

Structure and ADMIXTURE are model-based approaches widely used in hybridization studies to estimate ancestry coefficients for all samples in a formal statistical framework. The BI-based method structure infers population structure using genotype data consisting of unlinked markers by utilizing a Markov chain Monte Carlo (MCMC) algorithm to sample the relevant posterior distribution. The model assumes a user-specified number of ancestral populations in the sample (denoted by $\kappa$ ) and the individuals are probabilistically assigned to discrete populations or jointly to two or more populations if their genotypes indicate that they are admixed. The model in structure also assumes that every locus is in Hardy-Weinberg equilibrium and that there is linkage equilibrium within a population. The estimation procedure in ADMIXTURE is similar to 
12

structure, but it focuses on maximizing the likelihood rather than estimating the posterior, in an effort to enhance the speed of estimation to accommodate larger datasets.

Structure analyses were conducted using the admixture model. The length of the burn-in period and the number of MCMC replicates were set to $5 \times 10^{4}$ and $10^{5}$, respectively. There is no standardized way to estimate the minimum number of iterations needed for a given dataset, and while the number of iterations may vary between studies, our choices are appropriate considering the computational intensity required for multiple replicates. Moreover, the number of iterations used for burn-in and for drawing samples from the posterior in our analysis exceeds those used in previous comparison studies, including Oliveira et al. (2015) (105 iterations for burn-in and 106 sampled iterations) and Vähä and Primmer (2005) (10 ${ }^{4}$ iterations for burn-in and $5 \times 10^{4}$ sampled iterations). We manually chose $\kappa$ by examining the model networks (Fig. 1) as the number of distinct parental populations observed at the present time. For example, $\kappa=2$ was assumed for the single hybridization scenario (Fig. 1a) as it was expected to be composed of two parental populations and one hybrid population containing about half of the genetic information from each parent when $\gamma=0.5$. Likewise, we set $\kappa=\{2,4,6,4,2\}$ for the scenarios in Figure 1b-1f, respectively. While there are methods to estimate the number of genetic groups that best fits the data (e.g., STRUCTURE HARVESTER; Earl and vonHoldt, 2012), we bypassed this procedure to make as 'fair' comparisons as possible since we provided population information for $H y D e$ in the map file. We did not incorporate the ancestral population for the outgroup when selecting $\kappa$ because with only one sample for the outgroup, the method may not be able to accurately estimate population allele frequencies and in the worst case, it can disrupt the final result by unnecessarily allowing more than two ancestries for the admixed populations. Nevertheless, one extra structure analysis for the two-hybridization scenario (Fig. 1c) with $\kappa=5$ was conducted to assess the effect of allowing an extra population to accommodate the outgroup.

Lastly, ADMIXTURE analyses were conducted using the same $\kappa$ as structure 
analyses. Because ADMIXTURE requires the input to be in binary plink (.bed) format, only bi-allelic SNPs were included in the analyses. The analyses were terminated when the log-likelihood increased by $<10^{-4}$ between iterations. All analyses were carried out using the College of Arts and Sciences' high-performance computing cluster (Unity) at the Ohio State University. One hundred replicates were conducted for every parameter setting.

\section{Evaluation}

The power of a statistical test refers to the probability that the test rejects the null hypothesis $\left(H_{0}\right)$ when the alternative hypothesis is true. Here, only the performance of $H y D e$ and the $D$-statistic were compared in terms of their power to detect hybridization since structure and ADMIXTURE do not perform hypothesis testing. The power in HyDe and the $D$-statistic is defined as the proportion of the replicates for which $H_{0}: \gamma=0$ is rejected at an overall $\alpha=0.05$, with a Bonferroni correction applied to account for multiple testing. Specifically, $p$-values are adjusted by dividing 0.05 by the total number of triplets tested, which can be computed by $\frac{1}{2}(n)(n-1)(n-2)$ where $n$ is the number of ingroup populations identified in the map file. The power at $\gamma=0$ is equivalent to the type I error (TIE) rate. We also noted the FDR for both tests for the scenarios in Figure 1b-1f, which is the proportion of the erroneously rejected $H_{0}$ in the significant observations (Benjamini and Hochberg, 1995). The FDR is calculated by:

$$
F D R=\frac{V}{V+S}
$$

where $V$ refers to the number of significant incorrect triplets and $S$ is the number of significant correct triplets. The correct triplet refers to the true parent-hybrid relationship present in the model network topologies that explains a hybrid edge (Fig. 1), and the incorrect triplets refer to any other triplets. In this study, we denote a triplet by, for example, $\left(\mathrm{P}_{1}-\mathrm{H}-\mathrm{P}_{2}\right)$ for Figure 1a where the population $\mathrm{H}$ corresponds to a hybrid population and $\mathrm{P}_{1}$ and $\mathrm{P}_{2}$ represent the parents. We further relaxed the calculation of FDR for the $D$-statistic since the goal of the $D$-statistic is to detect the presence of 
14

introgression within the triplet, rather than to identify the hybrid-parent relationship. We subtracted the counts of triplets that contain at least one hybrid-parent pair, even if it only partially explains the true hybridization event, from the numerator of (7) thereby calculating a relaxed version of FDR for the $D$-statistic:

$$
r F D R=\frac{V-E}{V+S}
$$

where $E$ refers to the number of significant triplets that contain at least one pair of the hybrid-parent relationship. For the structure and ADMIXTURE analyses, we recorded the frequency with which hybrid populations were identified, noting that this measure of performance differs from the measurement of the power of a statistical test. Identifying hybrid individuals from the estimated ancestry coefficients is an inevitably subjective process; we declared an individual to be a hybrid if the estimated ancestry coefficient $\in(0.1,0.9)$.

The accuracy of the estimates of the parental contributions were evaluated by calculating the MSE of the estimated $\gamma$ from $H y D e$, the average of the inferred ancestry of individuals $(q i)$ in structure, and the ancestry fraction $(Q)$ values in ADMIXTURE. Note that $q i$ and $Q$ are denoted as $\gamma$ hereafter for convenience. The MSE is calculated by:

$$
M S E=\frac{1}{s} \sum_{i=1}^{s}\left(\hat{\gamma}_{i}-\gamma_{i}\right)^{2}
$$

where $s$ is the number of replicates (i.e., 100), $\hat{\gamma}_{i}$ is the estimate of $\gamma$ for dataset $i$, and $\gamma_{i}$ is the true value of $\gamma$ used to simulate the dataset $i$. The MSE can be decomposed into the sum of the variance and the square of the bias and hence low values of the MSE are indicative of estimators that are good in the sense that they minimize both variance and bias. The $D$-statistic was excluded from this comparison as it does not estimate $\gamma$.

Calculating the MSE was impractical for structure and ADMIXTURE results for the scenarios in Figure 1b-1f as more than two ancestries could be inferred for a hybrid. For these cases, we conducted Clustering Markov Packer Across K (CLUMPAK; Kopelman et al., 2015) analyses using the LargeKGreedy algorithm with default settings 
for the summation of the results. CLUMPAK was also used to eliminate the label switching phenomena in the structure output (Jakobsson and Rosenberg, 2007; Kopelman et al., 2015) and to reveal genuine multimodality. Multiple solutions were obtained in cases of multimodality, and we considered all distinct solutions in our analyses.

Benchmarks

All code used to complete the simulations and analyses and all output files and $\mathrm{R}$ scripts to reproduce the figures and table in this manuscript are available as Jupyter Notebook and R markdown documents on Dryad (doi: \#\#\#).

RESUlts Single Hybridization Scenarios

The power to detect hybridization for $H y D e$ and the $D$-statistic, as well as the frequency of hybrid identification in structure and ADMIXTURE, for different combinations of $\tau_{1}, \tau_{2}$, and $\gamma$ in the single hybridization scenarios (Fig. 1a) are summarized in Figure 2. HyDe performed very well in general regardless of the number of individuals per population used, though we noted that when a single individual per population was used, both the TIE rate and the power were slightly higher than when multiple individuals were included in the sample. The $D$-statistic detected hybrids with similarly high power with negligible TIE rates for most combinations of parameters $(\leqslant 0.06$, except for 0.19 when $\tau_{1}=0.5$ with $\tau_{2}=1.5$; Fig. 2a), in agreement with the results in Kubatko and Chifman (2019). HyDe did not perform well when ILS was prevalent when $\tau_{2}=0.05$ for all $\gamma$ and when $\tau_{2}=0.5$ for $\gamma=0.1$, where the power ranged from 0-0.1 (Fig. 2b). However, the power in $H y D e$ substantially increased to $\geqslant 0.95$ when we increased the dataset size by a factor of ten, although this was accompanied by a high TIE rate (Fig. 4a). Both detection power and accuracy of the estimates of $\gamma$ greatly improved $(\geqslant 0.97$ for $\gamma \geqslant 0.2)$ 

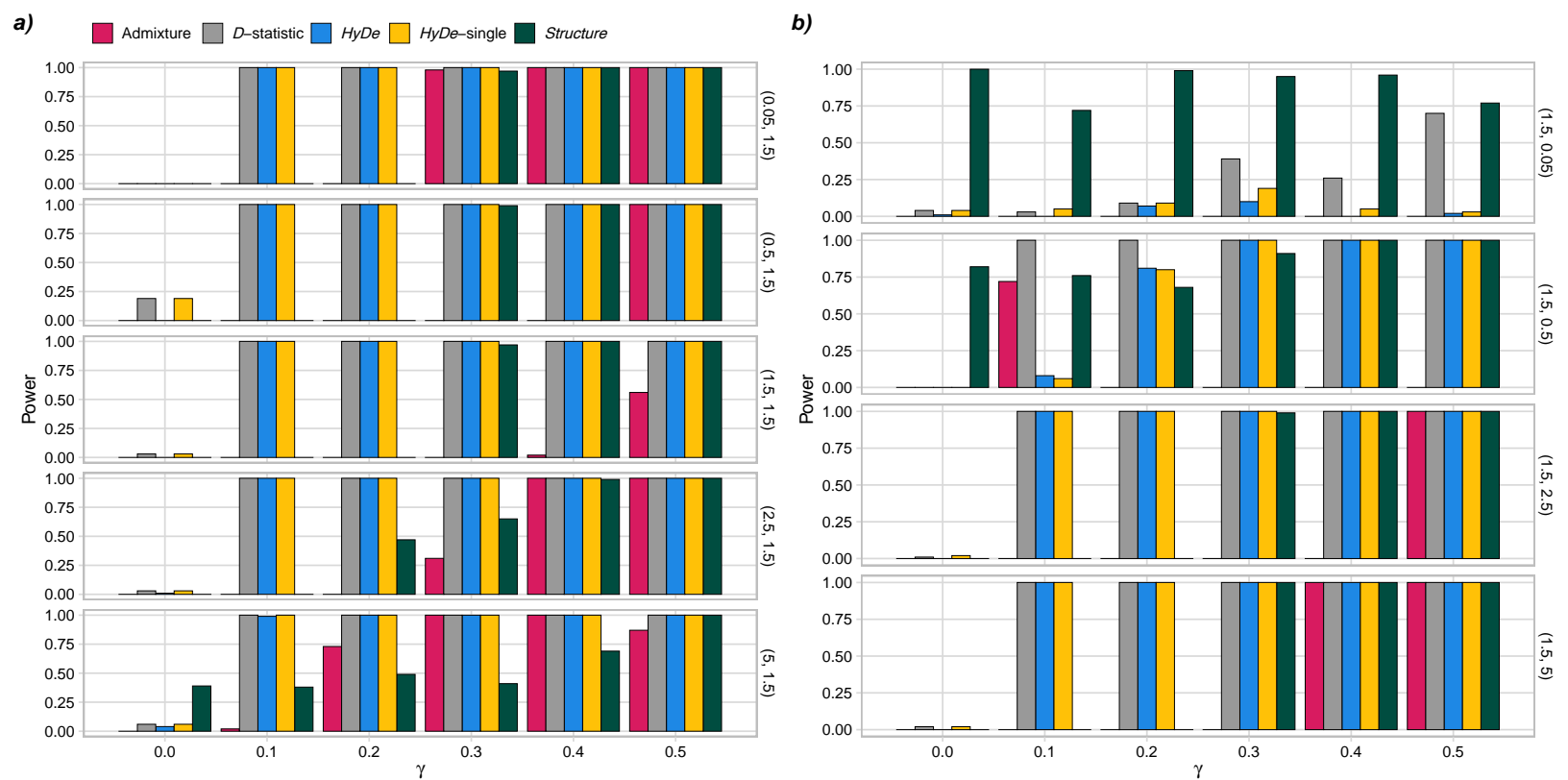

Fig. 2. The power to detect hybridization for the $D$-statistic and $H y D e(H y D e$-single refers to the power when a single individual per population is used) and the frequency of hybrid identification in ADMIXTURE and structure for different combinations of parental contributions, $\gamma \in\{0,0.1,0.2,0.3,0.4,0.5\}$, with a) $\tau_{1} \in\{0.05,0.5,1.5,2.5,5\}$ and $\tau_{2}$ fixed at 1.5 ; and b) $\tau_{1}$ fixed at 1.5 and $\tau_{2} \in\{0.05,0.5,2.5,5\}$ in single hybridization scenarios (Fig. 1a). The two numbers in parenthesis on right side of each plot specify $\left(\tau_{1}, \tau_{2}\right)$.

with no TIE when the size of the dataset was increased by a factor of 100 (Fig. 4b). The power for the $D$-statistic was also relatively poor $(0.03-0.7)$ in high ILS scenarios, however, it also recovered to 1 as $\tau_{2}$ was increased to 0.5 .

ADMIXTURE failed to identify any hybrid samples in many cases, particularly when the parental contributions were asymmetric and/or the amount of ILS was high, although one exception occurred when $\tau_{2}=0.5$ at $\gamma=0.1$. Similarly, structure did not perform well when $\gamma \leqslant 0.2$, with some exceptions when $\tau_{1} \geqslant 2.5$ or $\tau_{2} \leqslant 0.5$. Structure also showed high rates of false positives $(0.39-1)$ when $\tau_{1}<2.5$ or $\tau_{2} \leqslant 0.5$ where it detected admixed population at $\gamma=0$.

Figure 3 presents estimates of $\gamma$ and their MSE from ADMIXTURE, HyDe, and structure for different combinations of $\tau_{1}, \tau_{2}$, and $\gamma$ in the single hybridization scenario (Fig. 1a). For the estimates in ADMIXTURE, the frequency with which hybrids were identified was generally the highest for the symmetric parental contributions, though the 


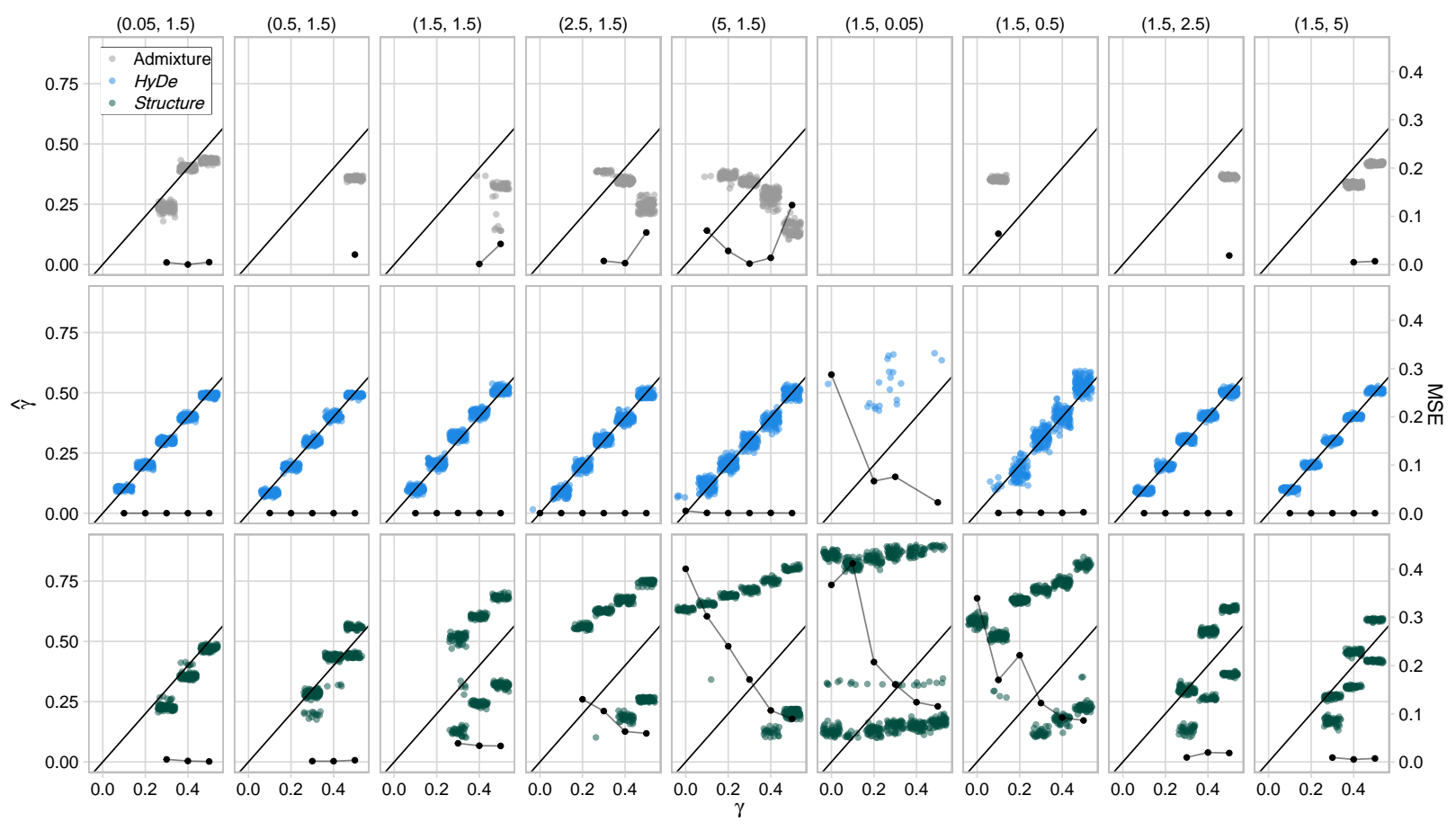

Fig. 3. Estimates of $\gamma$ (denoted as $\hat{\gamma}$ on the left y-axis) and the mean squared error (MSE; right y-axis) in ADMIXTURE, HyDe, and structure for the different combinations of parameters: $\tau_{1} \in\{0.05,0.5,1.5,2.5,5\}$ with $\tau_{2}$ fixed at 1.5 or $\tau_{1}$ fixed at 1.5 with $\tau_{2} \in\{0.05,0.5,2.5,5\}$ at $\gamma \in\{0,0.1,0.2,0.3,0.4,0.5\}$ in single hybridization scenarios (Fig. 1a). The two numbers in parenthesis on top specify $\left(\tau_{1}, \tau_{2}\right)$. The black solid line represents the expected $\gamma$ when MSE $=0$, the color-coded unconnected points represent the estimates of $\gamma$ for each tool, and the connected black line with points represents the MSE.

MSE was not always the smallest for $\gamma=0.5$. ADMIXTURE failed to identify hybrids in most cases when $\tau_{2}$ was varied where the observations were not sufficient to find a pattern in the method's performance. Similarly, even when structure identified hybrids when $\gamma \leqslant 0.2$ and $\tau_{1} \geqslant 2.5$ or $\tau_{2} \leqslant 0.5$, the estimates were relatively inaccurate as reflected by their high MSEs. In many cases, the structure estimates exhibited multimodality by clustering above and below the true $\gamma$, and the difference between the two clusters (i.e., modes) decreased as $\tau_{1}$ shortened or $\tau_{2}$ lengthened. While the absolute value of the structure estimates generally increased with $\gamma$, such a pattern was hard to find for high ILS scenarios. HyDe estimates $\gamma$ much more accurately than both structure and ADMIXTURE in all cases. Even when $H y D e$ performed poorly (e.g., for $\tau_{2}=0.05$ ), its estimates of $\gamma$ were more accurate than the estimates from structure. Moreover, the HyDe 
a)

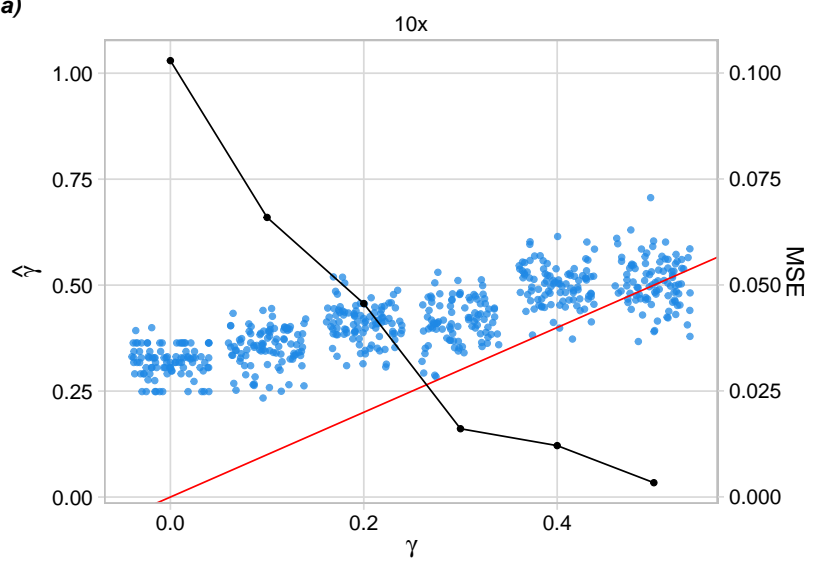

b)

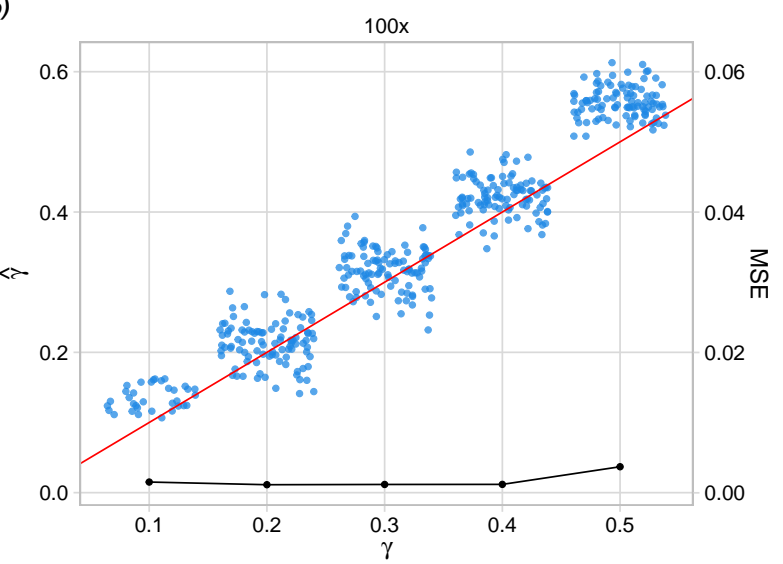

Fig. 4. Estimates of $\gamma$ (denoted by $\hat{\gamma}$ on the left y-axis) and the MSE (right y-axis) of HyDe for single hybridization scenarios (Fig. 1a) when $\tau_{1}=0.05$ and $\tau_{2}=1.5$ at $\gamma \in\{0,0.1,0.2,0.3,0.4,0.5\}$ when the dataset size was increased by a factor of a) ten (1,000 loci) and b) 100 (10,000 loci). The red solid line represents the expected $\gamma$ when MSE $=$ 0 , the unconnected blue points represent the estimates of $\gamma$, and the connected black line with points represents the MSE.

estimates for the high ILS scenario became more accurate as the number of loci increased (Fig. 4), illustrating that the inaccurate estimates were due to inadequate signal in the data rather than methodological incompetency.

\section{Introgression, Multi-hybridization, and Complex Hybridization Scenarios}

For the introgression, multi-hybridization, and mixture of recent and ancestral hybridization scenarios (Fig. 1b-1f), both $H y D e$ and the $D$-statistic exhibited a detection power of 1 for all correct triplets regardless of the number of individuals sampled from the populations. The FDR (7) for HyDe was generally negligible in all analyses except for the mixture of ancestral and recent hybridization events (Fig. 1f). The $D$-statistic showed very high FDR in all scenarios, ranging from 0.6 to 0.947 (Table 1), although these should be interpreted with caution. When the triplets that contain at least one pair of the hybrid and a parent were used to calculate the rFDR in (8), the rate substantially decreased, becoming as low as zero for the introgression and mixture of recent and ancestral hybridization scenarios. This is intuitive because any permutation of the populations in these scenarios will contain at least one hybrid-parent pair. In contrast, the rFDR for the 
Table 1. The false discovery rate (FDR) and the relaxed FDR ( $F F D R$ ) in HyDe and the $D$-statistic for the introgression, multi-hybridization and complex hybridization scenarios (Fig. 1b-1f).

\begin{tabular}{|c|c|c|c|c|}
\hline Hybridization Scenario & FDR-HyDe $e^{1}$ & FDR-HyDe-single ${ }^{2}$ & FDR-D & $\mathrm{rFDR}-D$ \\
\hline Introgression (Fig. 1b) & 0 & 0 & 0.667 & 0 \\
\hline Two independent events (Fig. 1c) & 0.015 & 0.043 & 0.9 & 0.3 \\
\hline Three independent events (Fig. 1d) & 0.007 & 0.013 & 0.947 & 0.316 \\
\hline Three hybridization events that overlap (Fig. 1e) & 0 & 0.004 & 0.69 & 0.228 \\
\hline A mixture of ancestral and recent events (Fig. 1f) & 0.5 & 0.501 & 0.6 & 0 \\
\hline
\end{tabular}

Note: ${ }^{1}$ multiple individuals per population were included; ${ }^{2}$ a single individual per population was included.

multi-hybridization scenarios remained high, ranging from 0.228 to 0.316 .

Figure 5a shows estimates of $\gamma$ and their MSE in HyDe and structure in the introgression scenario (Fig. 1b). While the two methods identified introgressants in all replicates, ADMIXTURE failed to detect any. The accuracy of the estimates in structure was good with MSE $<0.015$, although the estimates showed multimodality as was observed in the single hybridization scenarios. The estimates in $H y D e$ were more accurate than structure but with slightly larger MSE $(<0.009)$ than its estimates in the single hybridization scenarios. The HyDe estimates and the MSE for various timings of the introgression event and for various choices of $\gamma$ are illustrated in Figure 5b. The estimates were more accurate when introgression (node $u$ in Fig. 1b) occurred later than the split event (node $v$ ) and when the parental contributions were more asymmetric. However, the accuracy recovered for the more symmetric parental contributions as the distance between $u$ and $v$ was shortened.

For the multi- and complex hybridization scenarios (Fig. 1c-1f), estimates of $\gamma$ and the MSE in HyDe are shown in Figure 6. A graphical display of all distinct solutions of the ADMIXTURE and structure replicates identified via CLUMPAK analysis are presented in Figure 7. In the scenario with two recent hybridization events (Fig. 1c), HyDe unequivocally (i.e., no other triplet was found to be significant except the true ones) and accurately estimated $\gamma$ for both hybrid edges (Fig. 6a) with MSE $<0.001$. Assuming $\kappa=4$, CLUMPAK summarized ADMIXTURE replicates into two distinct solutions (i.e., modes; Kopelman et al., 2015) where one correctly identified both hybrid populations and their 
a)

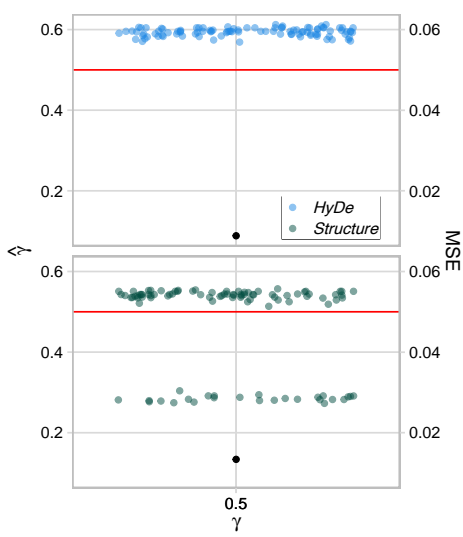

b)

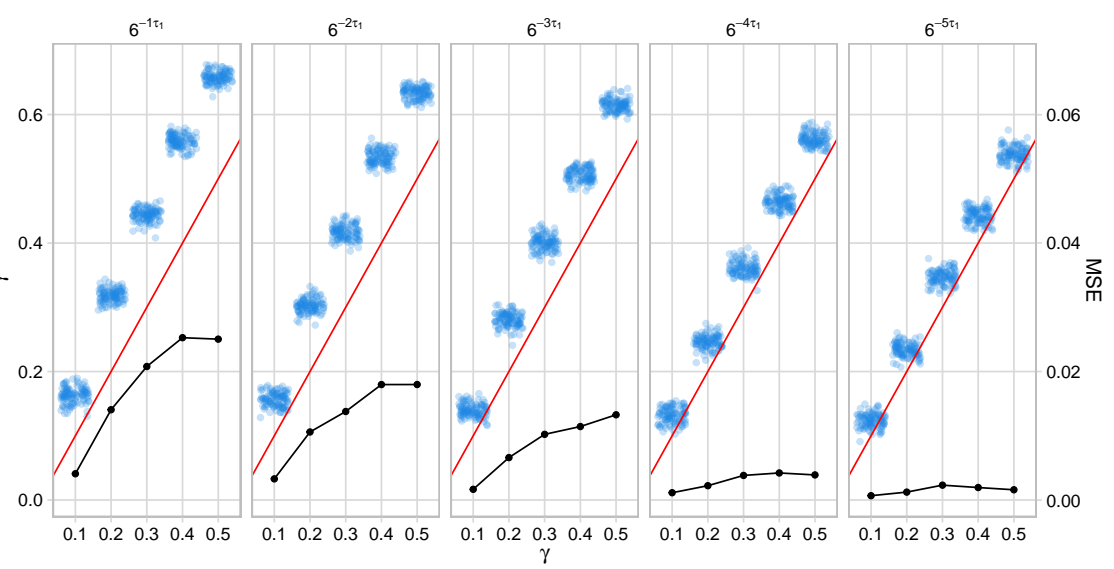

Fig. 5. a) Estimates of $\gamma$ and the MSE of HyDe (top) and structure (bottom) for the introgression scenario (Fig. 1b). b) Estimates of $\gamma$ and the MSE for the introgression scenario in HyDe for varying timings of the introgression event. Each plot is labelled at the top by the time of introgression that represents the position of the node $u$ in the model network. The time of introgression gets closer to $\tau_{1}$ from left to right. The connected or unconnected black points represent the MSE, and the red solid line represents the expected value of $\gamma$ when MSE $=0$.

parents with $\gamma \approx 0.35$ (Fig. 7a; contained $61 \%$ of replicates) whereas the other solution only identified $\mathrm{H}_{1}$ with $\gamma \approx 0.65$ (Fig. $7 \mathrm{~b}$; contained $39 \%$ of replicates). Structure replicates found three distinct solutions where two of them identified only one admixed population, either $\mathrm{H}_{1}$ or $\mathrm{H}_{2}$, with contributions from three ancestries that include both parents as well as the outgroup population in a ratio of approximately $0.38,0.38$, and 0.24 , respectively (Fig. 7c and 7e; contained $68 \%$ of replicates combined); however, one solution did not find any admixed population (Fig. 7d; contained $32 \%$ of replicates). When we repeated the structure analysis with $\kappa=5$ to prevent the outgroup contributing to the admixed population by assigning it as a distinct population, four distinct solutions were identified. Three out of the four solutions failed to identify any admixed population (i.e., ancestry coefficient $<0.1$; Fig. $7 \mathrm{f}-7 \mathrm{~h}$; contained $90 \%$ of replicates combined) but one solution that was supported by $10 \%$ of replicates identified both $\mathrm{H}_{1}$ and $\mathrm{H}_{2}$, but contributions from three ancestries that included two parents and one outgroup population persisted (Fig. 7i). The accuracy of $\gamma$ estimates in HyDe for the scenario with three recent independent hybridization events (Fig. 1d) was outstanding, with the MSE $<0.001$ for each of the three hybrid edges (Fig. 6b). Assuming $\kappa=6$, all ADMIXTURE replicates converged into a 


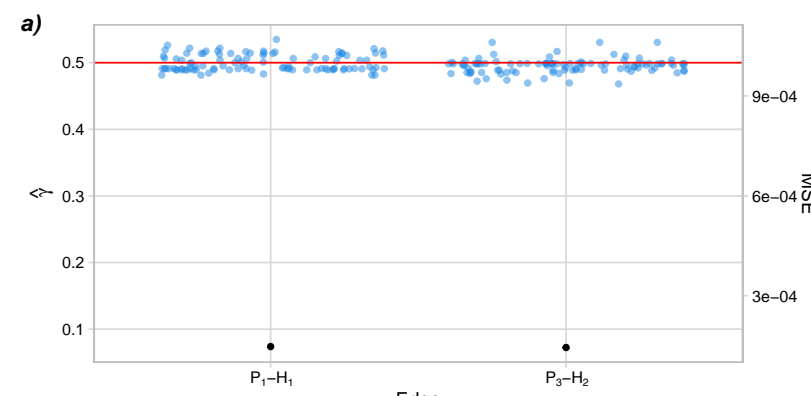

b)
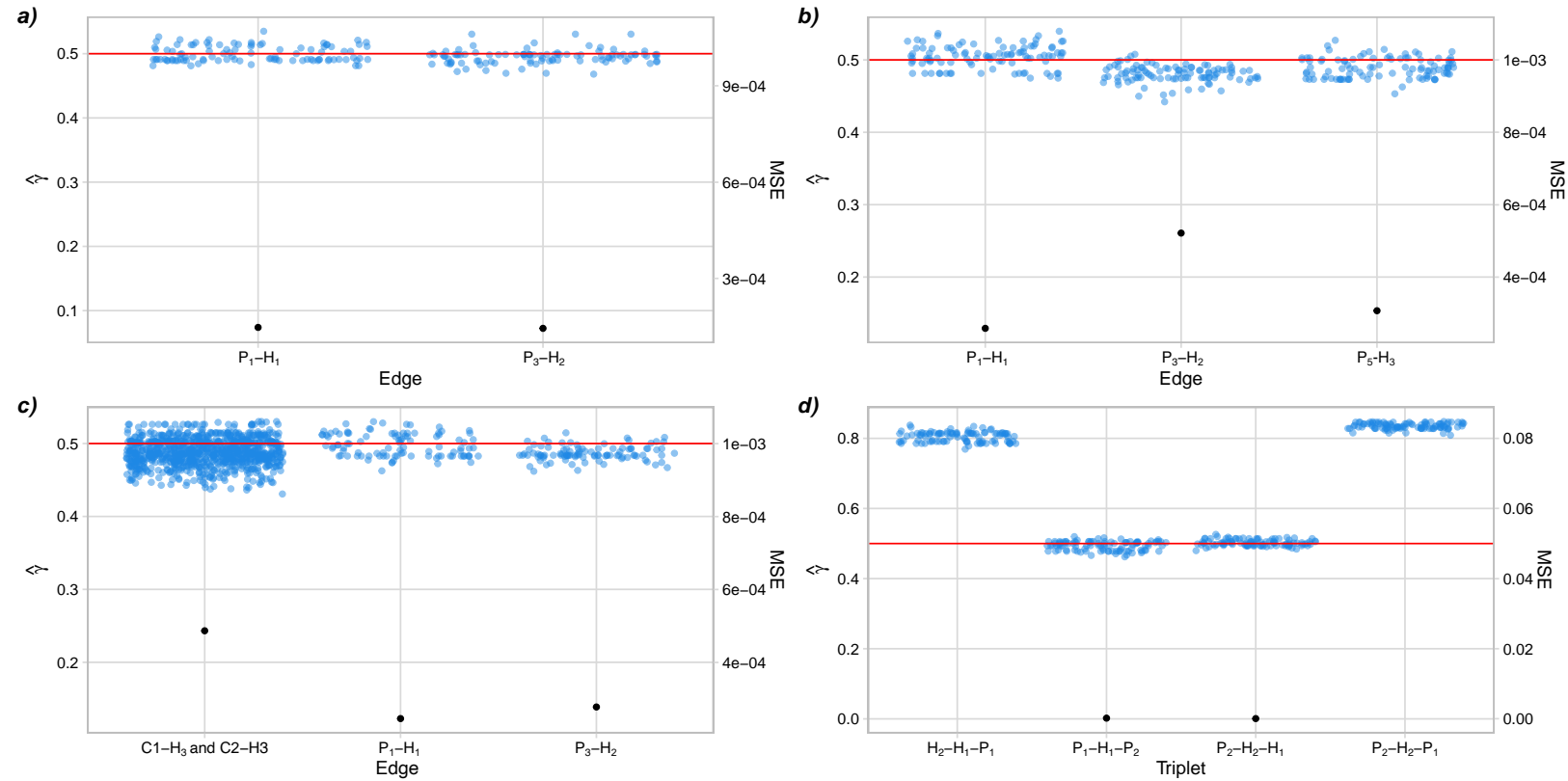

Fig. 6. Estimates of $\gamma$ and the MSE of HyDe for the a) two independent hybridization events, b) three independent hybridization events, c) three overlapping hybridization events, and d) the mixture of ancestral and recent hybridization events illustrated in Figures 1d-1f, respectively. The horizontal red line represents the true values of $\gamma$, and the black points represent the MSE for the corresponding hybrid edge. The blue points in a), b), and c) represent the estimates of $\gamma$ for the corresponding edge that links the parent-hybrid pair labelled in the $\mathrm{x}$-axis where $\mathrm{C} 1$ and $\mathrm{C} 2$ in c) represent any member in the clades $\left(\mathrm{P}_{1}, \mathrm{H}_{1}, \mathrm{P}_{2}\right)$ and $\left(\mathrm{P}_{3}, \mathrm{H}_{2}, \mathrm{P}_{4}\right)$ in Figure 1e, respectively. d) The estimates of parental contributions from the first population in the corresponding triplets labelled in the $\mathrm{x}$-axis to the second population.

single solution that identified only one of the three admixed populations, $\mathrm{H}_{3}$, with $\gamma \approx 0.35$ (Fig. 7j). Four distinct solutions were identified from the structure replicates where one solution that contained $51 \%$ of replicates (Fig. $7 \mathrm{k}$ ) did not identify any admixed population and each of the other three solutions identified a single admixed population (Fig. 7l-7n) that consists of four ancestries that includes both parents, the outgroup, and an ancestry that does not characterize any of the contemporary populations. The two parental populations, however, constituted the majority $(<0.9)$ for these admixed populations. In the scenario with an ancient hybridization event followed by two recent hybridization events (Fig. 1e), HyDe accurately estimated $\gamma$ with MSE $<0.001$ for all 11 correct triplets (Fig. 6c). Note that there are nine possible permutations that explain the parent-hybrid relationships for $\mathrm{H}_{3}$ where one of the parents is from the clade with $\left(\mathrm{P}_{1}, \mathrm{H}_{1}\right.$, $\left.\mathrm{P}_{2}\right)$ and the other is from the clade with $\left(\mathrm{P}_{3}, \mathrm{H}_{2}, \mathrm{P}_{4}\right)$. The ADMIXTURE outputs yielded 

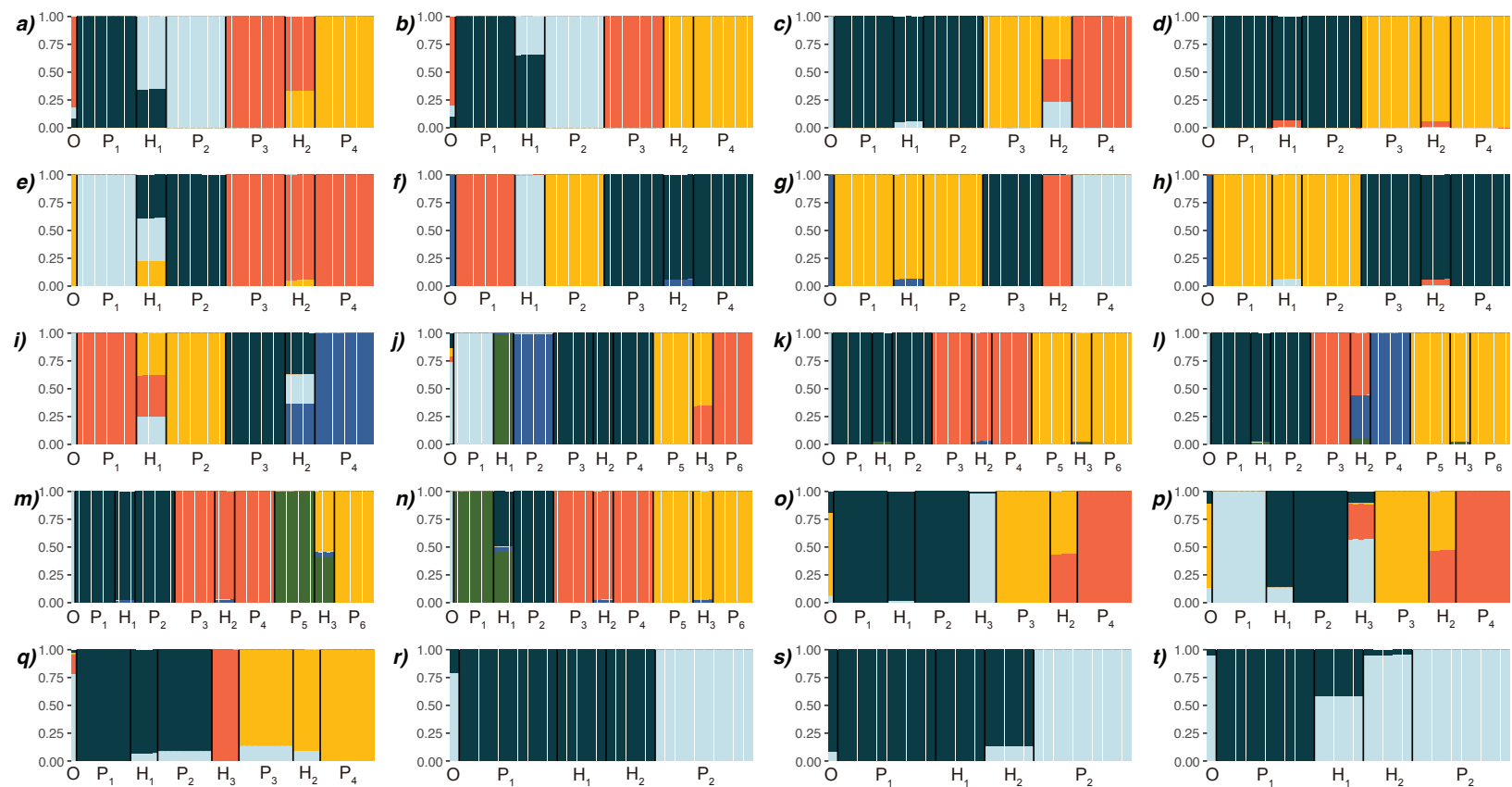

Fig. 7. Distinct Clustering Markov Packer Across K solutions for a-b) ADMIXTURE and c-e) structure analyses at $\kappa=4$ for the scenario with two independent hybridization events (Fig. 1c); f-i) structure analysis at $\kappa=5$ for the scenario with two independent hybridization events (Fig. 1c); j) ADMIXTURE and $\mathrm{k}-\mathrm{n}$ ) structure analyses at $\kappa=$ 6 for the scenario with three independent hybridization events (Fig. 1d); o-p) ADMIXTURE and q) structure analyses at $\kappa=4$ for the scenario with three overlapping hybridization events (Fig. 1e); and r) ADMIXTURE and $\mathrm{s}-\mathrm{t})$ structure analyses at $\kappa=2$ for the scenario with mixture of ancestral and recent hybridization (Fig. 1f). Each population is delimited by black line in the plot and labeled on x-axis; y-axis denotes ancestral coefficients.

two equally supported solutions for which one solution only identified one admixed population with $\gamma \approx 0.44$ (Fig. 7o) and the other identified all three admixed populations,

$\mathrm{H}_{1}$ with $\gamma=0.14, \mathrm{H}_{2}$ with $\gamma=0.44$, and $\mathrm{H}_{3}$ with contributions from all four parental populations where $\mathrm{P}_{1}$ and $\mathrm{P}_{4}$ composed the majority $(\approx 0.9 ;$ Fig. $7 \mathrm{p})$. The structure replicates identified a single solution with no admixture for any of the hybrid populations but with $\mathrm{P}_{3}$ incorrectly identified as admixed with $\gamma=0.14$ (Fig. 7q).

Finally, Figure 1f illustrates a scenario where the product of an ancestral hybridization event becomes the parent of a recent hybrid. HyDe accurately estimated $\gamma$ with MSE $<0.001$ for the two hybrid edges present in the model network. However, it also found significance for the incorrect triplets $\left(\mathrm{P}_{1}-\mathrm{H}_{1}-\mathrm{H}_{2}\right)$ and $\left(\mathrm{P}_{1}-\mathrm{H}_{2}-\mathrm{P}_{2}\right)$, though they are not directly observed in the network, with $\gamma \approx 0.19$ and 0.16 , respectively (Fig. $6 \mathrm{~d}$ ). The MSE for these unexpected triplets was not calculated as the true $\gamma$ for these triplets was 
unknown. The ADMIXTURE replicates converged to a single solution that did not identify any admixed populations (Fig. 7r). The Structure replicates were summarized into two distinct solutions where one identified $\mathrm{H}_{2}$ with $\gamma \approx 0.14$ but not $\mathrm{H}_{1}$ (Fig. 7s; contained $54 \%$ of replicates) and the other identified $\mathrm{H}_{1}$ with $\gamma \approx 0.42$ but not $\mathrm{H}_{2}$ (Fig. 7t; contained $46 \%$ of replicates).

We have illustrated that $H y D e$ is as powerful as the $D$-statistic in detecting hybridization with negligible FDR regardless of the number of individuals per population in the dataset. Also, estimates of $\gamma$ in $H y D e$ are more robust and accurate than the estimates in structure and ADMIXTURE in all hybridization scenarios considered in this study. HyDe clearly demonstrates an advantage over the $D$-statistic in that it can naturally accommodate multiple individuals per population, thereby allowing more information to be utilized in estimating site pattern probabilities leading to more accurate detection of hybridization (Blischak et al., 2018). In HyDe, site patterns for the population triplets (or quartet, when outgroup is included) are counted by considering all permutations of the individuals in each population. For example, the number of unique quartets for the four population $\left(\mathrm{O}-\mathrm{P}_{1}-\mathrm{H}-\mathrm{P}_{2}\right)$ would be number of individuals in outgroup $\left(N_{O}\right)$ $\times N_{P_{1}} \times N_{H} \times N_{P_{2}}$ for the single hybridization scenario (Fig. 1a), and the number of site patterns for these four populations would be simply the sum of the patterns appearing in all of the permutations of individuals. In contrast, the $D$-statistic usually considers a subset of this count by including a single individual per population, for example, it only counts site patterns from a single permutation by choosing one individual from each population in the quartet $\left(\mathrm{O}-\mathrm{P}_{1}-\mathrm{H}-\mathrm{P}_{2}\right)$. If the site pattern counts of multiple individuals from $\mathrm{HyDe}$ were to be directly used to calculate the $D$-statistic, then it would incorrectly find significance 
24

even in the absence of reticulation due to the amplified difference in $n_{A B B A}$ and $n_{B A B A}$.

Nevertheless, at least three factors can impact the performance of HyDe. First, an excessive amount of ILS can reduce both the detection power and the accuracy of the $\gamma$ estimates. The detection power in HyDe dropped from 0.99-1 to 0-0.19 (Fig. 2) when the length of $\tau_{2}$ was reduced to 0.05 in the single hybridization scenario (Fig. 1a). This is because the gene copy of an individual in the hybrid population, H, may fail to coalesce with the ortholog in the parent's genome (either $\mathrm{P}_{1}$ or $\mathrm{P}_{2}$, the one that contributed the gene to $\mathrm{H}$ ) within the very short interval between the split and hybridization event. This increases the probability that the gene copies from the two parents coalesce with each other before either coalesces with $\mathrm{H}$, producing a relationship that disagrees with the model network. Consequently, the probability of each of the three possible gene tree topologies for the three ingroup populations (i.e., $\left(P_{2},\left(P_{1}, H\right)\right) ;\left(P_{1},\left(P_{2}, H\right)\right)$; and $\left.\left(H,\left(P_{1}, P_{2}\right)\right)\right)$ will be roughly one-third. In short, ILS adds to both ABBA and BABA counts, diluting information for $H y D e$ and masking the footprint of hybridization within the hybrid's genome. As expected, the power of the method increases with the sample size (Fig. 4), reconfirming that large datasets may be necessary for the optimal performance for HyDe when there is a high level of ILS.

Second, ancient hybridization (more precisely, an increase in the ratio of $\tau_{1}$ to $\tau_{2}$ ) can reduce the accuracy of the $\gamma$ estimates. Assuming the substitution rate is homogeneous across the tree, it is intuitive that taxa with longer external branch lengths will have more time to accumulate mutations and the site patterns that would otherwise be informative for hybrid detection can be lost. Fortunately, our results indicate that the effect of increased $\tau_{1}$ on the estimates is not a major concern, as the largest MSE at $\tau_{1}=5$ was $<0.001$ (Fig. 3). However, it is important to note that ancient hybridization is not uncommon in empirical systems (e.g., Genner and Turner, 2012; Escudero et al., 2014; Sun et al., 2015; Li et al., 2016; Schumer et al., 2016) and it is likely that estimating $\gamma$ from the sampled genome would be more complex than from our simulated data. Even if 
the mutation rate for these populations were small, populations with longer external branches are particularly challenging due to their unique evolutionary histories during this time, including factors such as ongoing gene flow shortly after the divergence, distinct histories of introgression, maintenance of assortative mating, and rate heterogeneity (Sankararaman et al., 2014; Cahill et al., 2015).

Third, we argue that for scenarios involving introgression (Fig. 1b), as opposed to hybrid speciation (Fig. 1a), the time and direction of introgression must be taken into account for the accurate estimation of $\gamma$. The hybridization model assumed in $H y D e$ is time-consistent (i.e., both parents of the hybrid node must coexist in time; Cardona et al., 2009), an assumption that is violated in cases of introgressive hybridization (see Fig. 1b; the vertical position of nodes $u$ and $v$ are different). The introgression scenario in Figure $1 \mathrm{~b}$ does not represent direct hybridization between $u$ and $v$ at $z$, but illustrates our best inference of evolutionary history based on the data in present time. Based on the network, we can postulate the product of hybridization between $u$ and the ancestor of $\mathrm{I}_{1}$ that coexisted with $u$ is not extant anymore but is completely merged with $\mathrm{I}_{1}$ via backcrossing. In this case, because alleles in $\mathrm{I}_{1}$ contributed by $\mathrm{P}_{1}$ experienced less time to mutate than the alleles shared with $\mathrm{P}_{2}$, the site pattern $\mathrm{ABBA}$ is more likely to persist than AABB, thereby inhibiting accurate estimation of $\gamma$ (recall (4), (5), and (6)), as evident in the larger than expected $\hat{\gamma}$ values in Figure 5b.

Moreover, a biological system can sometimes harbor both parents, a hybrid, and a backcrossing population whose evolutionary relationships can be illustrated as in Figure if (e.g., Wolfe et al., 1998). This scenario would also be what we might observe if the hybrid between $u$ and the ancestor of $\mathrm{I}_{1}$ that coexisted with $u$ did not go extinct and continued to backcross with $\mathrm{I}_{1}$. In this system, complete sampling can unintentionally complicate the interpretation of $H y D e$ results because each hybrid population can have two significant parent-hybrid relationships that are not mutually exclusive. For example, in Figure 1f, emergence of $\mathrm{H}_{1}$ can be correctly explained by hybridization between $\mathrm{P}_{1}$ and $\mathrm{P}_{2}$ with 
26

$\gamma=0.5$ or incorrectly between $\mathrm{H}_{2}$ and $\mathrm{P}_{1}$ with approximately $80 \%$ genomic contribution from $\mathrm{H}_{2}$. Similarly, $\mathrm{H}_{2}$ can have either $\mathrm{P}_{2}$ and $\mathrm{H}_{1}$ or $\mathrm{P}_{2}$ and $\mathrm{P}_{1}$ as parents also with $\gamma \approx 0.8$ for the hybrid edge between $\mathrm{H}_{2}$ and $\mathrm{P}_{2}$ (Fig. 6d). It would be impossible to identify the correct triplet if the true relationship is unknown, and such significance for incorrect triplets could result in a misinterpretation of the evolutionary history of these taxa.

Our observations imply the need for a method that distinguishes hybrid speciation from introgression given the information on the site pattern frequencies. In fact, the idea of phylogenetic invariants hints at a potentially promising method. For example, note that Figure 1a can be decomposed into topologies (1) and (2) and that we will get the exact same topologies even when the positions of parents are switched. Then, the probability of the site pattern frequencies for the triplets when computed for $\left(\mathrm{P}_{1}-\mathrm{H}_{-} \mathrm{P}_{2}\right)$ or $\left(\mathrm{P}_{2}-\mathrm{H}-\mathrm{P}_{1}\right)$ should be equal. On the other hand, if the same procedure is done for Figure 1b, the site pattern frequencies counted for the two triplets will not be expected to be equal because the two decomposed trees will have the same topology but with different branch lengths. While this idea should work well when $\gamma=0.5$ and the time of introgression is sufficiently distant from the speciation event, it might not be as powerful as expected when the parental contribution is not symmetric. Progress toward a similar goal has recently been made. Hibbins and Hahn (2019) made an important contribution by proposing statistical models that can be used to test hypotheses relating to the timing and the direction of introgression that potentially allow one to distinguish hybrid speciation from introgression, although application of the method may be premature since it involves strong assumptions that may not hold for empirical datasets.

\section{On the Use of Population Clustering Methods for Hybrid Detection}

Model-based clustering algorithms have been extremely useful in population genetic studies. Running on datasets consisting of a few loci to those with thousands of genetic markers, these methods have been shown to be powerful in classifying individual genotypes 
into subpopulations, a biological level at which conventional phylogenetic methods often fail to identify a resolved structure among individuals. The probabilistic quantities estimated for every individual - the ancestry coefficients - are often evolutionarily interpreted as the proportion of that individual's alleles that are inherited from a specific ancestral population.

It is important to stress that the identification of hybrids based on the ancestry coefficient is prone to subjectivity. In practice, hybrid individuals are identified by setting parametric boundaries for the 'pure' populations (i.e., non-admixed populations). It is common practice to select these thresholds arbitrarily; for example, many studies define the pure population by a collection of individuals with ancestry coefficient greater than 0.8 (or 0.9) for a single ancestral population while some studies use a higher cut-off (e.g., 0.95 or even 0.99; Steeves et al., 2010). Lowering the threshold for the non-admixed populations is often justified in order to prevent overestimating the number of hybrid samples. This is a paradoxical argument because it will inevitably lead to the overestimation of the number of pure populations, particularly in case of skewed parental contributions. Sometimes the thresholds are decided post hoc in order to produce a desired population structure that incorporates the previous identification of individuals based on other criteria (e.g., morphology). More often, no clear justification for the choice of threshold is provided.

Even if the thresholds were appropriately justified, an individual with an estimated ancestry coefficient that is intermediate between two pure populations may not necessarily have arisen via historical hybridization. An intermediate ancestry coefficient could be observed even in the absence of hybridization when there is a large genetic divergence between populations or an excessive amount of ILS in the dataset. The former case, for instance, is observed when $\tau_{1}=5$ in Figure 1a where structure identified population $\mathrm{H}$ as admixed at $\gamma=0$ (Fig. 3). In this case, even though the populations $\mathrm{H}$ and $\mathrm{P}_{2}$ share the MRCA, and are expected to have originated from the same ancestral genetic pool, the length of $\tau_{1}$ causes the two populations to become differentiated enough that the method 
28

cannot confidently cluster them together. However, this is less likely to be an issue in practice if $\kappa$ can be statistically estimated as three, instead of two, as assumed in our analyses. The latter case is more problematic, because ILS and hybridization may produce similar footprint in an organism's genome and are difficult to distinguish unless variation in the evolutionary histories of the underlying genes is taken into account, a process which is not incorporated in the models underlying the population clustering methods (Carstens et al., 2013). For example, the ancestry coefficients estimated in structure for the population $\mathrm{H}$ when $\tau_{2}=0.05$ in Figure $1 \mathrm{a}$ at $\gamma=0$ were not distinguishable from other cases with same length of $\tau_{2}$ when $\gamma \neq 0$. This result also illustrates that the excessive amount of ILS can override signals of hybridization in structure analysis. The inability to distinguish ILS from hybridization is a crucial limitation for use of population assignment methods in hybridization studies, as hybridization often occurs within closely related, rapidly evolving systems where pre- or post-zygotic barriers are not yet fully established.

Moreover, the absence of admixed populations is not evidence of the absence of hybridization for the population clustering methods. Both ADMIXTURE and structure often failed to identify hybrids when the parental contribution was asymmetric, while the severity varied depending on the length of $\tau_{1}$ or $\tau_{2}$. An asymmetric contribution from the parents to the hybrid is not uncommon in nature, as exemplified by Heliconius heurippa where the genome contains parental contributions in the ratio of 0.825 to 0.125 (Mavárez et al., 2006). Additional examples of asymmetric hybridization can be easily found in plants (Tiffin et al., 2001), invertebrates (Coyne and Orr, 1989; Presgraves, 2002), and vertebrates (Malone and Fontenot, 2008). In theses cases, hybrids are likely to be missed by the population clustering methods. Even with symmetric parental contributions, ADMIXTURE completely failed to identify introgressants and both ADMIXTURE and structure rarely identified all hybrid populations in the multi-hybridization scenarios. In these cases, historical hybridization in empirical systems will be underestimated despite efforts for comprehensive data collection. 
Finally, it is difficult to use estimates of the ancestry coefficient to infer the hybrid status of an individual with certainty. In some studies, the numerical values of the ancestry coefficients were used to categorize admixed individuals into $\mathrm{F}_{1}, \mathrm{~F}_{2}$, or introgressant populations. Detailed interpretation of ancestry coefficients is not recommended, not only because they may not be accurate, as evident in the high MSE in our simulations, but also because different processes can result in identical ancestry coefficients. For example, Lawson et al. (2018) explored an issue in ADMIXTURE and structure that produces indistinguishable plots in both the presence or absence of admixture when recent genetic drift is strong or the process deviates in other ways from the underlying inference model. Furthermore, estimates of the ancestry coefficients can result in multiple distinct solutions even when the same dataset is used, known as genuine multimodality, particularly among methods that involve stochastic simulation in the algorithm such as structure (Jakobsson and Rosenberg, 2007; Kopelman et al., 2015). Genuine multimodality was prevalent in our structure estimates, with replicates often forming two or more clusters in a single hybridization scenario and multiple sets of solutions in more complex scenarios.

Nevertheless, population clustering methods can be extremely useful in detecting hybrid populations if employed in conjunction with $H y D e$. HyDe requires information on the assignment of individuals into discrete populations, however, such information may not be always readily available for empirical datasets. In this case, population clustering analysis prior to running $H y D e$ can provide important information on the number of genetically distinct populations (although accurate estimation of $\kappa$ would still be crucial) and assignments of individuals into those populations. Moreover, results from the population clustering methods and HyDe may be complementary to each other in hypothesizing historical evolutionary processes. For example, a dataset may contain a high amount of ILS if an admixed population was found by the clustering methods but none of the triplets found significance for hybridization in $H y D e$, or a population may experienced multiple introgression events that result in asymmetric genomic contributions from the 
REFERENCES

parents if significance was observed using $H y D e$ but no admixed samples were identified in the clustering analyses.

\section{CONCLUSions}

Interspecific hybridization plays an important role in nature by promoting or preventing evolutionary divergence between organisms and is being widely recognized as an essential process to understand the evolution of taxa across the Tree of Life. While accurate detection of hybrids is also a crucial step in practice (for instance, in conservation management), our results suggest that the popularly employed population clustering methods may not be reliable for such task and may potentially result in inappropriate conclusions about natural processes. Alternatively, we observe promising potential for site pattern based methods for hybrid detection that outcompete the population clustering methods in terms of both power and accuracy of their estimates of $\gamma$, although they may require large-scale genomic datasets to perform optimally under complex evolutionary scenarios. Finally, this study implies the need for phylogenetic network inference methods that can overcome scalability issues to simultaneously detect hybrids as well as their evolutionary history.

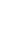


REFERENCES

Alexander, D. H., J. Novembre, and K. Lange. 2009. Fast model-based estimation of ancestry in unrelated individuals. Genome Research 19:1655-1664.

Anderson, E. 1953. Introgressive hybridization. Biological Reviews 28:280-307.

Anderson, E. C. and K. K. Dunham. 2008. The influence of family groups on inferences made with the program Structure. Molecular Ecology Resources 8:1219-1229.

Anderson, E. C. and E. A. Thompson. 2002. A model-based method for identifying species hybrids using multilocus genetic data. Genetics 160:1217-1229.

Barilani, M., A. Sfougaris, A. Giannakopoulos, N. Mucci, C. Tabarroni, and E. Randi. 2007. Detecting introgressive hybridisation in rock partridge populations (Alectoris graeca) in Greece through Bayesian admixture analyses of multilocus genotypes. Conservation Genetics 8:343-354.

Benjamini, Y. and Y. Hochberg. 1995. Controlling the false discovery rate: A practical and powerful approach to multiple testing. Journal of the Royal Statistical Society: Series B (Methodological) 57:289-300.

Blischak, P. D., J. Chifman, A. D. Wolfe, and L. S. Kubatko. 2018. HyDe: A python package for genome-scale hybridization detection. Systematic Biology 67:821-829.

Bohling, J. H., J. R. Adams, and L. P. Waits. 2013. Evaluating the ability of Bayesian clustering methods to detect hybridization and introgression using an empirical red wolf data set. Molecular Ecology 22:74-86.

Cahill, J. A., I. Stirling, L. Kistler, R. Salamzade, E. Ersmark, T. L. Fulton, M. Stiller, R. E. Green, and B. Shapiro. 2015. Genomic evidence of geographically widespread effect of gene flow from polar bears into brown bears. Molecular Ecology 24:1205-1217. 
Cardona, G., M. Llabres, F. Rossello, and G. Valiente. 2009. Metrics for phylogenetic networks I: Generalizations of the Robinson-Foulds metric. IEEE/ACM Transactions on Computational Biology and Bioinformatics 6:46-61.

Carstens, B. C., T. A. Pelletier, N. M. Reid, and J. D. Satler. 2013. How to fail at species delimitation. Molecular Ecology 22:4369-4383.

Chan, K. O., C. R. Hutter, P. L. Wood, L. Lee Grismer, and R. M. Brown. 2019. Species delimitation in the grey zone: introgression obfuscates phylogenetic inference and species boundaries in a cryptic frog complex (Ranidae: Pulchrana picturata). bioRxiv .

Chang, C. C., C. C. Chow, L. C. Tellier, S. Vattikuti, S. M. Purcell, and J. J. Lee. 2015. Second-generation PLINK: Rising to the challenge of larger and richer datasets. GigaScience 4:7.

Corander, J. and P. Marttinen. 2006. Bayesian identification of admixture events using multilocus molecular markers. Molecular Ecology 15:2833-2843.

Coyne, J. A. and H. A. Orr. 1989. Patterns of speciation in Drosophila. Evolution $43: 362-381$.

Degnan, J. H. and N. A. Rosenberg. 2006. Discordance of species trees with their most likely gene trees. PLoS Genetics 2:e68.

Durand, E. Y., N. Patterson, D. Reich, and M. Slatkin. 2011. Testing for ancient admixture between closely related populations. Molecular Biology and Evolution 28:2239-2252.

Earl, D. A. and B. M. vonHoldt. 2012. STRUCTURE HARVESTER: A website and program for visualizing STRUCTURE output and implementing the Evanno method. Conservation Genetics Resources 4:359-361.

Elworth, R. A. L., C. Allen, T. Benedict, P. Dulworth, and L. K. Nakhleh. 2018. Dgen: A test statistic for detection of general introgression scenarios. in WABI. 
Escudero, M., D. A. Eaton, M. Hahn, and A. L. Hipp. 2014. Genotyping-by-sequencing as a tool to infer phylogeny and ancestral hybridization: A case study in Carex (Cyperaceae). Molecular Phylogenetics and Evolution 79:359-367.

Felsenstein, J. 1991. Counting phylogenetic invariants in some simple cases. Journal of Theoretical Biology 152:357-376.

Folk, R. A., P. S. Soltis, D. E. Soltis, and R. Guralnick. 2018. New prospects in the detection and comparative analysis of hybridization in the tree of life. American Journal of Botany 105:364-375.

Genner, M. J. and G. F. Turner. 2012. Ancient hybridization and phenotypic novelty within Lake Malawi's Cichlid Fish radiation. Molecular Biology and Evolution 29:195-206.

Gerard, D., H. L. Gibbs, and L. Kubatko. 2011. Estimating hybridization in the presence of coalescence using phylogenetic intraspecific sampling. BMC Evolutionary Biology $11: 291$.

Goulet, B. E., F. Roda, and R. Hopkins. 2017. Hybridization in plants: Old ideas, new techniques. Plant Physiology 173:65-78.

Green, R. E., J. Krause, A. W. Briggs, T. Maricic, U. Stenzel, M. Kircher, N. Patterson, H. Li, W. Zhai, M. H. Y. Fritz, N. F. Hansen, E. Y. Durand, A. S. Malaspinas, J. D. Jensen, T. Marques-Bonet, C. Alkan, K. Prufer, M. Meyer, H. A. Burbano, J. M. Good, R. Schultz, A. Aximu-Petri, A. Butthof, B. Hober, B. Hoffner, M. Siegemund, A. Weihmann, C. Nusbaum, E. S. Lander, C. Russ, N. Novod, J. Affourtit, M. Egholm, C. Verna, P. Rudan, D. Brajkovic, Z. Kucan, I. Gusic, V. B. Doronichev, L. V. Golovanova, C. Lalueza-Fox, M. de la Rasilla, J. Fortea, A. Rosas, R. W. Schmitz, P. L. F. Johnson, E. E. Eichler, D. Falush, E. Birney, J. C. Mullikin, M. Slatkin, R. Nielsen, J. Kelso, M. Lachmann, D. Reich, and S. Paabo. 2010. A draft sequence of the neandertal genome. Science 328:710-722. 
Hermansen, J. S., S. A. Saether, T. O. Elgvin, T. Borge, E. Hjelle, and G.-P. Saetre. 2011.

Hybrid speciation in sparrows I: Phenotypic intermediacy, genetic admixture and barriers to gene flow. Molecular Ecology 20:3812-3822.

Hibbins, M. S. and M. W. Hahn. 2019. The timing and direction of introgression under the multispecies network coalescent. Genetics 211:1059-1073.

Holder, M. T., J. A. Anderson, and A. K. Holloway. 2001. Difficulties in detecting hybridization. Systematic Biology 50:978-982.

Hudson, R. R. 2002. Generating samples under a Wright-Fisher neutral model of genetic variation. Bioinformatics 18:337-338.

Isomura, N., K. Iwao, and H. Fukami. 2013. Possible natural hybridization of two morphologically distinct species of Acropora (Cnidaria, Scleractinia) in the pacific: Fertilization and larval survival rates. PLoS ONE 8:e56701.

Ito, H., T. Langenhorst, R. Ogden, and M. Inoue-Murayama. 2015. Population genetic diversity and hybrid detection in captive zebras. Scientific Reports 5:13171.

Jakobsson, M. and N. A. Rosenberg. 2007. CLUMPP: A cluster matching and permutation program for dealing with label switching and multimodality in analysis of population structure. Bioinformatics 23:1801-1806.

Joly, S., P. A. McLenachan, and P. J. Lockhart. 2009. A statistical approach for distinguishing hybridization and incomplete lineage sorting. The American Naturalist 174:E54-E70.

Jónsson, H., M. Schubert, A. Seguin-Orlando, A. Ginolhac, L. Petersen, M. Fumagalli, A. Albrechtsen, B. Petersen, T. S. Korneliussen, J. T. Vilstrup, T. Lear, J. L. Myka, J. Lundquist, D. C. Miller, A. H. Alfarhan, S. A. Alquraishi, K. A. S. Al-Rasheid, J. Stagegaard, G. Strauss, M. F. Bertelsen, T. Sicheritz-Ponten, D. F. Antczak, E. Bailey, R. Nielsen, E. Willerslev, and L. Orlando. 2014. Speciation with gene flow in 
equids despite extensive chromosomal plasticity. Proceedings of the National Academy of Sciences 111:18655-18660.

Kalinowski, S. T. 2011. The computer program STRUCTURE does not reliably identify the main genetic clusters within species: Simulations and implications for human population structure. Heredity 106:625-632.

Khosravi, R., H. R. Rezaei, and M. Kaboli. 2013. Detecting hybridization between Iranian Wild Wolf (Canis lupus pallipes) and free-ranging domestic dog (Canis familiaris) by analysis of microsatellite markers. Zoological Science 30:27-34.

Kopelman, N. M., J. Mayzel, M. Jakobsson, N. A. Rosenberg, and I. Mayrose. 2015. CLUMPAK : A program for identifying clustering modes and packaging population structure inferences across K. Molecular Ecology Resources 15:1179-1191.

Kubatko, L. S. 2009. Identifying hybridization events in the presence of coalescence via model selection. Systematic Biology 58:478-488.

Kubatko, L. S. and J. Chifman. 2019. An invariants-based method for efficient identification of hybrid species from large-scale genomic data. BMC Evolutionary Biology 19:112.

Kumar, V., F. Lammers, T. Bidon, M. Pfenninger, L. Kolter, M. A. Nilsson, and A. Janke. 2017. The evolutionary history of bears is characterized by gene flow across species. Scientific Reports 7:46487.

Latch, E. K., G. Dharmarajan, J. C. Glaubitz, and O. E. Rhodes. 2006. Relative performance of Bayesian clustering software for inferring population substructure and individual assignment at low levels of population differentiation. Conservation Genetics 7:295-302.

Lawson, D. J., L. van Dorp, and D. Falush. 2018. A tutorial on how not to over-interpret STRUCTURE and ADMIXTURE bar plots. Nature Communications 9:3258. 
Lehtinen, R. M., A. F. Steratore, M. M. Eyre, E. S. Cassagnol, M. L. Stern, and H. A. Edgington. 2016. Identification of widespread hybridization between two terrestrial salamanders using morphology, coloration, and molecular markers. Copeia 104:132-139.

Li, G., B. W. Davis, E. Eizirik, and W. J. Murphy. 2016. Phylogenomic evidence for ancient hybridization in the genomes of living cats (Felidae). Genome Research 26:1-11.

Lischer, H. E. L. and L. Excoffier. 2012. PGDSpider: An automated data conversion tool for connecting population genetics and genomics programs. Bioinformatics 28:298-299.

Maddison, W. P. 1997. Gene trees in species trees. Systematic Biology 46:523-536.

Malinsky, M. 2019. Dsuite - fast d-statistics and related admixture evidence from vcf files. bioRxiv .

Mallet, J. 2005. Hybridization as an invasion of the genome. Trends in Ecology and Evolution 20:229-237.

Malone, J. H. and B. E. Fontenot. 2008. Patterns of reproductive isolation in toads. PLoS ONE 3:e3900.

Martin, M. D., F. G. Vieira, S. Y. Ho, N. Wales, M. Schubert, A. Seguin-Orlando, J. B. Ristaino, and M. T. P. Gilbert. 2016. Genomic characterization of a south american Phytophthora hybrid mandates reassessment of the geographic origins of Phytophthora infestans. Molecular Biology and Evolution 33:478-491.

Mavárez, J., C. A. Salazar, E. Bermingham, C. Salcedo, C. D. Jiggins, and M. Linares. 2006. Speciation by hybridization in Heliconius butterflies. Nature 441:868-871.

Meng, C. and L. S. Kubatko. 2009. Detecting hybrid speciation in the presence of incomplete lineage sorting using gene tree incongruence: A model. Theor Popul Biol $75: 35-45$. 
Neophytou, C. 2014. Bayesian clustering analyses for genetic assignment and study of hybridization in oaks: Effects of asymmetric phylogenies and asymmetric sampling schemes. Tree Genetics \& Genomes 10:273-285.

Olave, M. and A. Meyer. 2020. Implementing large genomic SNP datasets in phylogenetic network reconstructions: A case study of particularly rapid radiations of cichlid fish. Systematic Biology Page syaa005.

Oliveira, R., E. Randi, F. Mattucci, J. D. Kurushima, L. A. Lyons, and P. C. Alves. 2015. Toward a genome-wide approach for detecting hybrids: Informative SNPs to detect introgression between domestic cats and European wildcats (Felis silvestris). Heredity 115:195-205.

Page, A. J., B. Taylor, A. J. Delaney, J. Soares, T. Seemann, J. A. Keane, and S. R. Harris. 2016. SNP-sites: Rapid efficient extraction of SNPs from multi-FASTA alignments. Microbial Genomics 2.

Patterson, N., P. Moorjani, Y. Luo, S. Mallick, N. Rohland, Y. Zhan, T. Genschoreck, T. Webster, and D. Reich. 2012. Ancient admixture in human history. Genetics 192:1065-1093.

Pauers, M. J., K. R. Fox, R. A. Hall, and K. Patel. 2018. Selection, hybridization, and the evolution of morphology in the Lake Malawi endemic cichlids of the genus Labeotropheus. Scientific Reports 8:15842.

Pease, J. B. and M. W. Hahn. 2015. Detection and polarization of introgression in a five-taxon phylogeny. Systematic Biology 64:651-662.

Piry, S., A. Alapetite, J.-M. Cornuet, D. Paetkau, L. Baudouin, and A. Estoup. 2004. GeneClass2: A software for genetic assignment and first-generation migrant detection. Journal of Heredity 95:536-539. 
Presgraves, D. C. 2002. Patterns of postzygotic isolation in Lepidoptera. Evolution $56: 1168-1183$.

Pritchard, J. K., M. Stephens, and P. Donnelly. 2000. Inference of population structure using multilocus genotype data. Genetics 155:945-959.

Rambaut, A. and N. C. Grassly. 1997. Seq-Gen: An application for the Monte Carlo simulation of DNA sequence evolution along phylogenetic trees. Bioinformatics $13: 235-238$.

Randi, E. 2008. Detecting hybridization between wild species and their domesticated relatives. Molecular Ecology 17:285-293.

Sankararaman, S., S. Mallick, M. Dannemann, K. Prüfer, J. Kelso, S. Pääbo, N. Patterson, and D. Reich. 2014. The genomic landscape of Neanderthal ancestry in present-day humans. Nature 507:354-357.

Sanz, N., R. M. Araguas, R. Fernández, M. Vera, and J.-L. García-Marín. 2009. Efficiency of markers and methods for detecting hybrids and introgression in stocked populations. Conservation Genetics 10:225-236.

Schumer, M., R. Cui, D. L. Powell, G. G. Rosenthal, and P. Andolfatto. 2016. Ancient hybridization and genomic stabilization in a swordtail fish. Molecular Ecology 25:2661-2679.

Solís-Lemus, C. and C. Ané. 2016. Inferring phylogenetic networks with Maximum Pseudolikelihood under incomplete lineage sorting. PLOS Genetics 12:e1005896.

Soraggi, S., C. Wiuf, and A. Albrechtsen. 2018. Powerful inference with the D-statistic on Low-Coverage Whole-Genome Data. G3 8:551-566.

Steeves, T. E., R. F. Maloney, M. L. Hale, J. M. Tylianakis, and N. J. Gemmell. 2010. Genetic analyses reveal hybridization but no hybrid swarm in one of the world's rarest birds: Hybridization without a hybrid swarm in Kaki. Molecular Ecology 19:5090-5100. 
Sun, M., D. E. Soltis, P. S. Soltis, X. Zhu, J. G. Burleigh, and Z. Chen. 2015. Deep phylogenetic incongruence in the angiosperm clade Rosidae. Molecular Phylogenetics and Evolution 83:156-166.

Tiffin, P., S. Olson, and L. C. Moyle. 2001. Asymmetrical crossing barriers in angiosperms. Proceedings of the Royal Society of London. Series B: Biological Sciences 268:861-867.

Twyford, A. D. and R. A. Ennos. 2012. Next-generation hybridization and introgression. Heredity 108:179-189.

Vähä, J.-P. and C. R. Primmer. 2005. Efficiency of model-based Bayesian methods for detecting hybrid individuals under different hybridization scenarios and with different numbers of loci. Molecular Ecology 15:63-72.

Wagner, N. D., L. He, and E. Hörandl. 2019. Relationships and genome evolution of polyploid salix species revealed by rad sequencing data. bioRxiv .

Wolfe, A. D., Q.-Y. Xiang, and S. R. Kephart. 1998. Diploid hybrid speciation in Penstemon (Scrophulariaceae). Proceedings of the National Academy of Sciences 95:5112-5115.

Yu, Y., J. Dong, K. J. Liu, and L. Nakhleh. 2014. Maximum likelihood inference of reticulate evolutionary histories. Proceedings of the National Academy of Sciences 111:16448-16453.

Zhang, B.-W., L.-L. Xu, N. Li, P.-C. Yan, X.-H. Jiang, K. E. Woeste, K. Lin, S. S. Renner, D.-Y. Zhang, and W.-N. Bai. 2019. Phylogenomics reveals an ancient hybrid origin of the Persian Walnut. Molecular Biology and Evolution 36:2451-2461.

Zheng, Y. and A. Janke. 2018. Gene flow analysis method, the D-statistic, is robust in a wide parameter space. BMC Bioinformatics 19:10. 